\title{
Absorption and Gain Spectra of Optically Excited Semiconductor Quantum Wires:
}

\section{Effects of Coulomb Correlation and Screening}

\author{
Ping Huai* , Tetsuo Ogawa \\ CREST, JST and Department of Physics, Osaka University, Toyonaka, Osaka \\ 560-0043, Japan
}

\begin{abstract}
We present a systematic study on optical spectra of one-dimensional semiconductor quantum wires by solving the semiconductor Bloch equations. Many-body Coulomb interactions are substantially enhanced by strong geometrical confinement in quantum wire systems. Our theoretical calculation reveals that phase-space filling has strong influence on the absorption spectra. Increasing carrier density bleaches the exciton peak in low-density regime, and induces considerable optical gain in very high density. Band-gap renormalization is partially cancelled by reduction of exciton binding energy, which increases as the cross-section of quantum wire decrease.
\end{abstract}

Key words: Semiconductor Quantum Wire, Coulomb Interaction, Quantum Confinement, Absorption and Gain Spectra, Semiconductor Bloch Equations, Phase-Space Filling 
Many-body Coulomb interactions play a key role in optical properties of highly-photoexcited low-dimensional semiconductors [1]. Under strong optical pumping, plasma screening of the Coulomb interaction causes significant reduction of exciton binding energy. However it has been observed that the exciton peak seems to be at a constant energy independent of carrier density [2]. Such constant exciton peak arises from the cancellation between band-gap renormalization (BGR) and reduction of binding energy [3].

When the dimensionality is lowered, the plasma screening of Coulomb interactions is less effective because the field line passing through the barrier material cannot be screened. Therefore the influence of static screening in quasi-onedimensional (1D) is considerably weaker than that in two/three dimensions, and the effect of phase-space filling becomes more important in quantum wires $[4]$.

Because of strong quantum confinement, the Coulomb interactions in quantum wires become quite complicated since electron/hole states are quantized in the lateral direction forming multiple subbands. A few analytical results $[5,6]$ have been achieved for the Coulomb interactions in semiconductor quantum wires. Most studies on optical properties of quantum wires are still based on Coulomb potentials obtained by using roughly approximated envelop functions or idealized geometries. Studies on exciton behaviors in quantum wire with realistic geometry, e.g., T-shaped GaAs quantum wires [7], are often restricted to zero carrier-density limit, i.e., the noninteracting exciton picture.

\footnotetext{
* Corresponding author: Ping Huai; Postal Address: Department of Physics, Osaka University, Machikaneyama 1-1, Toyonaka, Osaka 560-0043, Japan. Tel: +81 66850 5735; Fax: +81 668505351.

Email address: huai@acty.phys.sci.osaka-u.ac.jp (Ping Huai).
} 
There has been increasing experimental interest in 1D semiconductor devices, which are believed to have better performance than three dimensional ones due to concentration of density of states at the low energy regime. Highquality T-shaped quantum wires [8] have been fabricated with highly developed nanofabrication techniques.

Thus an aim of this study is to investigate the optical properties of quantum wires with realistic quantum confinement. Furthermore the effect of phasespace filling is clarified by systematic studies on T-shaped, cylindrical, and ribbon-like quantum wires. T-shaped quantum wires are discussed in this paper as an example. The Coulomb potentials $V_{k}^{i, j}$ are evaluated by averaging the $3 \mathrm{D}$ Coulomb interaction over the lateral envelop functions $\phi_{i}(x, y)$ and $\phi_{j}(x, y)$ :

$$
\begin{aligned}
V_{k}^{i, j}= & \frac{2 e^{2}}{\epsilon_{0}} \int_{-\infty}^{+\infty} d x_{1} \int_{-\infty}^{+\infty} d y_{1} \int_{-\infty}^{+\infty} d x_{2} \int_{-\infty}^{+\infty} d y_{2} \\
& \left|\phi_{i}\left(x_{1}, y_{1}\right)\right|^{2} K_{0}\left(k \sqrt{\left(x_{1}-x_{2}\right)^{2}+\left(y_{1}-y_{2}\right)^{2}}\right)\left|\phi_{j}\left(x_{2}, y_{2}\right)\right|^{2}
\end{aligned}
$$

Here $i$ and $j$ are the indices of the subbands, $k$ is the momentum in the wire direction, and $K_{0}(x)$ is the modified Bessel function of the second kind. Typical parameters for bulk GaAs are applied in the study: background dielectric constant $\epsilon_{0}=13.74$, the effective mass of electron (hole) $m_{e}=0.0665 m_{0}$, $m_{h}=0.457 m_{0}$. We also introduce Bohr radius $a_{0}=12.5 \mathrm{~nm}$ and exciton binding energy $E_{0}=4.2 \mathrm{meV}$ of bulk GaAs as the unit of length and energy, respectively.

The cross-section of a T-shaped quantum wire is shown in Fig. 1 (a). The lateral confinement is assumed to be infinite while carrier is free in the $z$ direction perpendicular to $x-y$ plane. Thus the wavefunctions of electrons 
and holes are of the same form irrespective of their effective-mass difference.

To obtain single particle wavefunctions, we solve the Schrödinger equation numerically taking into account the T-shaped confinement. Figure 1(b) shows the probability of the ground state of a T-shaped quantum wire with $L_{x}=$ $L_{y}=0.5 a_{0}$. The density is concentrated in the intersection of the two quantum wells, and decays exponentially out of the center regime in both arm well and stem well.

The Coulomb potentials is then calculated by using Eq. (1) for carriers in the ground state $(i=j=1)$. Results are shown in Fig. 2 for four symmetric T-shaped quantum wires with different cross-section size. As the lateral confinement becomes stronger, the Coulomb interaction is significantly enhanced. In the long-wavelength limit $k a_{0} \rightarrow 0$, the Coulomb potentials all approach to the limit $-\frac{2 e^{2}}{\epsilon_{0}} \ln (k)+$ const. It is easy to understand this logarithmic divergence if one notices $K_{0}(x) \rightarrow-\ln (x)$ if $x \ll 1$. In the short-wavelength limit, the Coulomb potentials of T-shaped quantum wire approach to zero in a manner different from those in 3D $\left(\propto k^{-2}\right)$ and $2 \mathrm{D}\left(\propto k^{-1}\right)$.

We consider a two-band model coupled with electromagnetic field $E(t)$ through dipole interaction,

$$
\begin{aligned}
H & =\sum_{k}\left(e_{e, k} \alpha_{k}^{\dagger} \alpha_{k}+e_{h, k} \beta_{-k}^{\dagger} \beta_{-k}\right) \\
& +\frac{1}{2} \sum_{k, k^{\prime}, q \neq 0} V_{q}\left(\alpha_{k+q}^{\dagger} \alpha_{k^{\prime}-q}^{\dagger} \alpha_{k^{\prime}} \alpha_{k}+\beta_{k+q}^{\dagger} \beta_{k^{\prime}-q}^{\dagger} \beta_{k^{\prime}} \beta_{k}-2 \alpha_{k+q}^{\dagger} \beta_{k^{\prime}-q}^{\dagger} \beta_{k^{\prime}} \alpha_{k}\right) \\
& -\sum_{k} E(t)\left(d_{c v} \alpha_{k}^{\dagger} \beta_{-k}^{\dagger}+\text { H.c. }\right)
\end{aligned}
$$

in which $\alpha_{k}^{\dagger}\left(\alpha_{k}\right)$ and $\beta_{k}^{\dagger}\left(\beta_{k}\right)$ are the creation (annihilation) operators of electrons in a conduction band and holes in a valence band, respectively. The momentum dependence is neglected in the optical dipole matrix element $d_{c v}$. 
The energies of electron and hole are given by $e_{e, k}=\frac{\hbar^{2} k^{2}}{2 m_{e}}+E_{g}^{0}$ and $e_{h, k}=\frac{\hbar^{2} k^{2}}{2 m_{h}}$, in which $E_{g}^{0}$ is the energy gap without BGR. We apply the Coulomb potential $V_{k}\left(\equiv V_{k}^{11}\right)$ shown in Fig. 2, which are the same for electron-electron, hole-hole, and electron-hole interactions due to infinite confinement.

Under the quasi-equilibrium condition, interband polarization $P_{k}(t)$ follows the semiconductor Bloch equations

$$
\begin{aligned}
{\left[i \hbar \frac{\partial}{\partial t}-\tilde{e}_{e, k}-\tilde{e}_{h, k}\right] P_{k}(t) } & =-\left[1-f_{e, k}-f_{h, k}\right] \Omega_{k}(t)+\left.\frac{\partial P_{k}(t)}{\partial t}\right|_{\text {scatt }} \\
\tilde{e}_{i, k} & =e_{i, k}-\sum_{k^{\prime} \neq k} V_{s,\left|k-k^{\prime}\right|} f_{i, k^{\prime}}, \quad i=e, h
\end{aligned}
$$

where $\tilde{e}_{i, k}$ is the renormalized kinetic energy of electron or hole. Here $V_{s, k}=$ $V_{k} / \epsilon(k, \omega)$ is the screened potential. Quasi-equilibrium condition implies that intraband relaxations are much faster than interband optical processes so that carrier distributions have already relaxed to the thermal Fermi distributions $f_{e, k}\left(f_{h, k}\right)$. The generalized Rabi frequency is defined by

$$
\Omega_{k}(t)=d_{c v} E(t)+\sum_{k^{\prime}} V_{s,\left|k-k^{\prime}\right|} P_{k^{\prime}}(t)
$$

The scattering term in Eq. (3) is important in the low-density regime where optical properties are strongly modified by the exciton-exciton scattering, biexciton formation, and dynamical screening. These many-body correlations are clarified by complicated quantum approaches, e.g., dynamics-controlled truncation or nonequilibrium Green's function theory. In the high-density regime, the Coulomb interaction becomes less important with respect to large kinetic energies of carriers. The scattering terms can be neglected, and Hartree-Fock approximation (HF) together with random phase approximation gives a good description of coherent optical processes. It is worth to examine the validity 
of HF theory before more sophisticated theory is applied. At the HF level, the Eq. (3) is reduced to the integral equation of the susceptibility function $\chi_{k}$,

$$
\begin{aligned}
& \chi_{k}=\chi_{k}^{0}\left(1+\frac{1}{d_{c v}} \sum_{k^{\prime}} V_{s,\left|k-k^{\prime}\right|} \chi_{k^{\prime}}\right), \\
& \chi_{k}^{0}=-d_{c v} \frac{1-f_{e, k}-f_{h, k}}{\hbar \omega-\tilde{e}_{e, k}-\tilde{e}_{h, k}+i \gamma},
\end{aligned}
$$

in which a phenomenological damping $\gamma\left(=0.05 E_{0}\right.$ in this study) is introduced to take into account the spectra broadening due to carrier-carrier and carrierphonon scattering.

We solve Eq. (5) by numerical matrix inversion technique with discrete momenta of Gaussian quadrature. Static screening of Coulomb interactions is neglected in the calculation since it is less important than the phase-space filling in the $1 \mathrm{D}$ system. The resulting complex susceptibility is given by $\chi(\omega)=\frac{1}{L} \sum_{k} d_{c v}^{\star} \chi_{k}$.

Figure 3 shows the absorption and gain spectra (imaginary part of $\chi(\omega)$ ) for a T-shaped wire with $L_{x}=L_{y}=0.5 a_{0}$ at $80 \mathrm{~K}$. The free exciton peak $\left(n a_{0}=0\right)$ is at about $3.52 E_{0}$ as shown in the inset. Another weaker peak located at $0.4 E_{0}$ is ascribed to the first excited state $(2 \mathrm{~s})$ of exciton. At finite density $n a_{0}=0.5$, the exciton peak shows a small redshift $\left(\approx 0.1 E_{0}\right)$ with respect to the large BGR $\left(\approx 3.4 E_{0}\right)$. In left-hand-side of Eq. $(3)$, the BGR is included in the diagonal part of polarization $P_{k}(t)$ as $\sum_{k^{\prime} \neq k} V_{s,\left|k-k^{\prime}\right|}\left(f_{e, k^{\prime}}+f_{h, k^{\prime}}\right) P_{k}(t)$. This term is compensated by the off-diagonal part in the right-hand-side $\left(-1+f_{e, k}+\right.$ $\left.f_{h, k}\right) \sum_{k^{\prime}} V_{s,\left|k-k^{\prime}\right|} P_{k^{\prime}}(t)$ that contributes to the binding energy of the exciton. Thus the BGR is partially cancelled by the binding energy reduction resulting from increasing carrier density. In quasi 1D systems, the phase-space filling $\left(-1+f_{e, k}+f_{h, k}\right)$ plays a more important role than the static screening. 
At higher density $n a_{0}=0.7$, the exciton peak is bleached while a build-up of optical gain appears. That indicated that exciton absorption has been replaced by the gain of exciton-hole-plasma. The gain is enhanced and broadened in even higher density $n a_{0}=0.9$. The dependence of gain spectra on the crosssection size is shown in Fig. 4 for density $n a_{0}=0.7$ at $T=80 \mathrm{~K}$. Four symmetric T-shaped quantum wires are investigated ranging from $0.5 a_{0}$ to $1.25 a_{0}$. As the size of the cross section decreases, the optical gain increases, and the maximum of gain is shifted to the lower energy side.

In this paper, optical spectra are discussed for T-shaped quantum wires with different size and carrier density. However, the energy difference is not taken into account for pure GaAs arm well, slightly doped stem well and heavily doped barriers. Further studies will be carried out for more realistic finite confinement in GaAs T-shaped quantum wires. Those results together with temperature-dependence will be discussed in details elsewhere.

\section{References}

[1] H. Haug and S. W. Koch, Quantum Theory of the Optical and Electronic Properties of Semiconductors (World Scientific, Singapore, 2004).

[2] W. Wegscheider, L. N. Pfeiffer, M. M. Dignam, A. Pinczuk, K. W. West, S. L. McCall, and R. Hull, Phys. Rev. Lett. 71 (1993) 4071.

[3] S. D. Sarma and D. W. Wang, Phys. Rev. Lett. 84 (2000) 2010.

[4] S. Benner and H. Haug, Europhys. Lett. 16 (1991) 579.

[5] W. Y. Lai and S. Das Sarma, Phys. Rev. B 33 (1986) 8874.

[6] A. Gold and A. Ghazali, Phys. Rev. B 41 (1990) 7626. 
[7] M. H. Szymanska, P. B. Littlewood, and R. J. Needs, Phys. Rev. B 63 (2001) 205317.

[8] M. Yoshita, H. Akiyama, L. N. Pfeiffer, K. W. West, Appl. Phys. Lett. 81 (2002) 49 . 
Fig. 1. (a) Cross section of T-shaped quantum wire. (b) Probability of single particle confined in a quantum wire with $L_{x}=L_{y}=0.5 a_{0}$.

Fig. 2. Coulomb potentials of T-shaped quantum wire with various cross-section size.

Fig. 3. Absorption and gain spectra as a function of the carrier density of T-shaped quantum wires with $L_{x}=L_{y}=0.5 a_{0}$. Parameters are set as $T=80 \mathrm{~K}$ and $\gamma=0.05 E_{0}$

Fig. 4. Gain spectra as a function of the cross-section size of T-shaped quantum wire. Carrier density is $n a_{0}=0.7$. Parameters are set as $T=80 \mathrm{~K}$ and $\gamma=0.05 E_{0}$. 
(a)

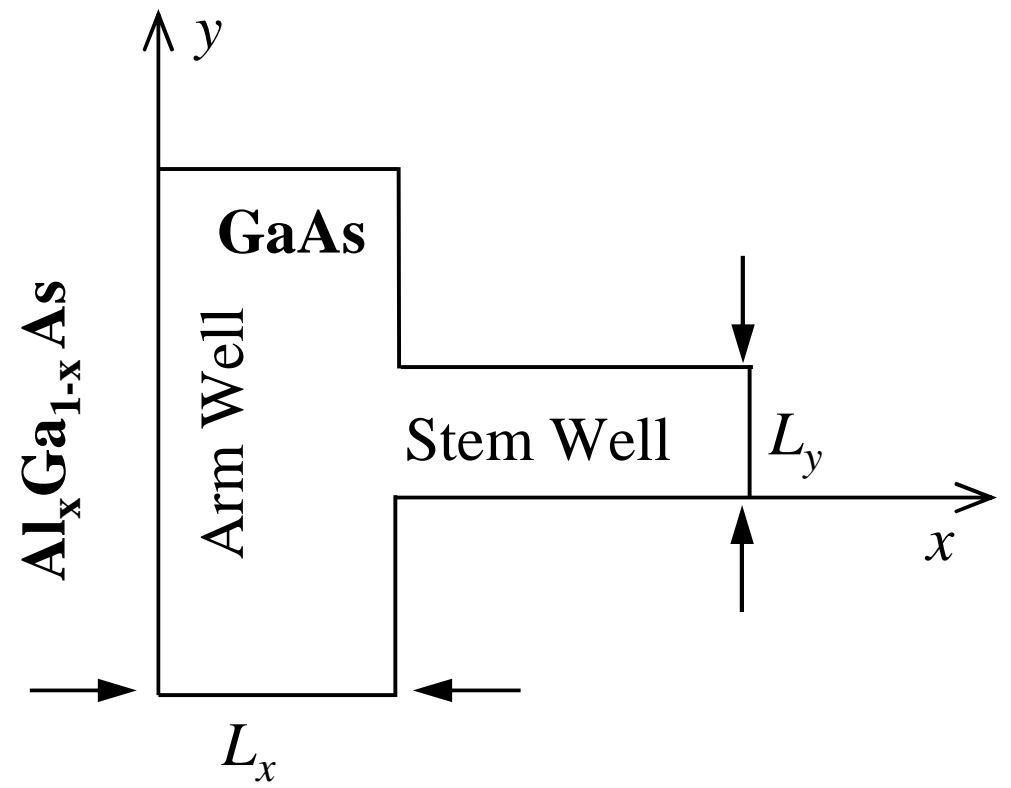

(b)

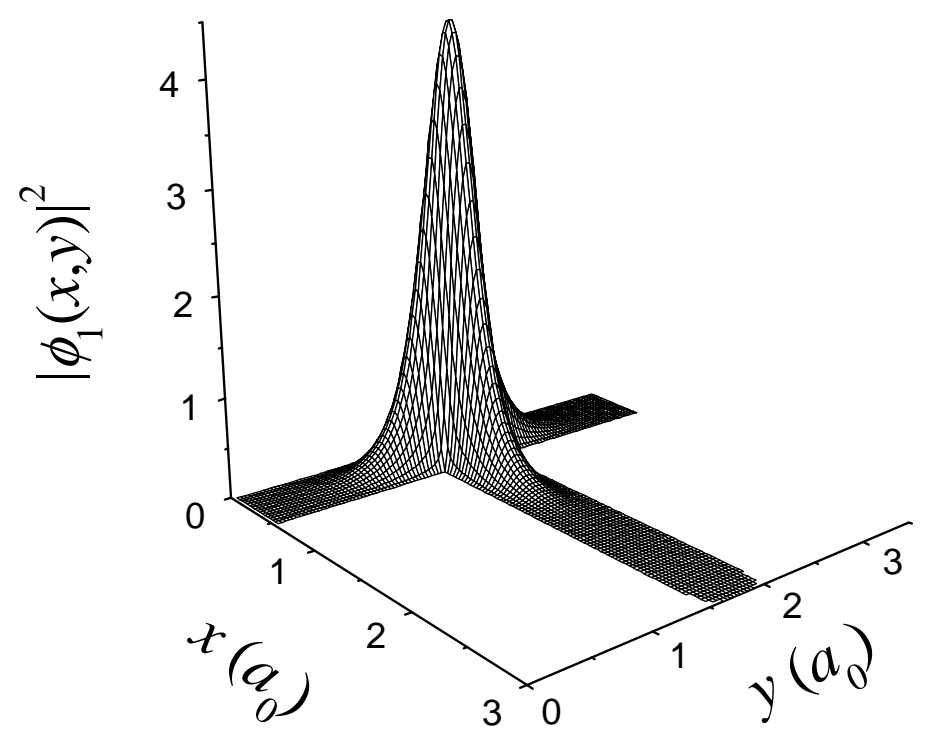

Fig.1 


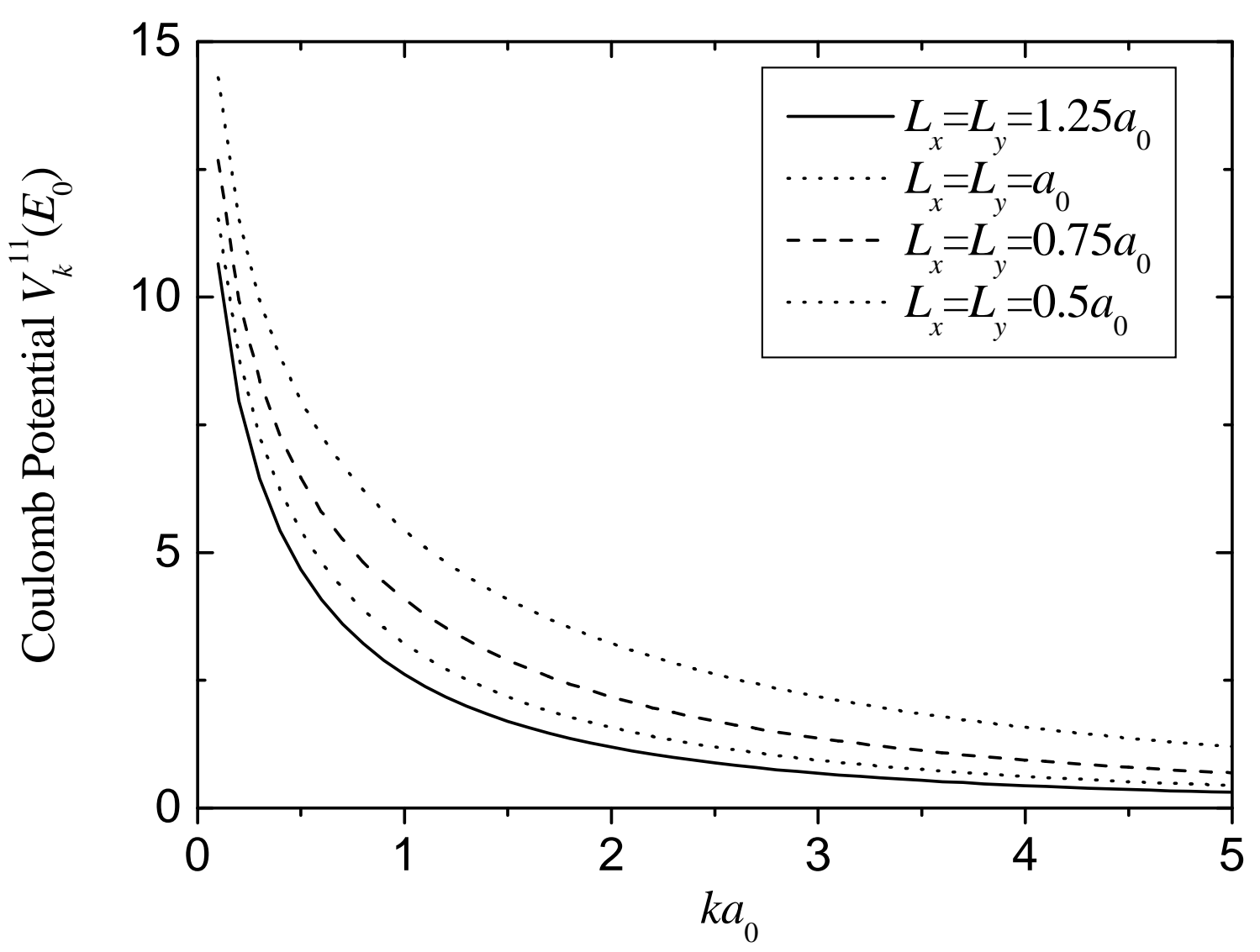

Fig.2 


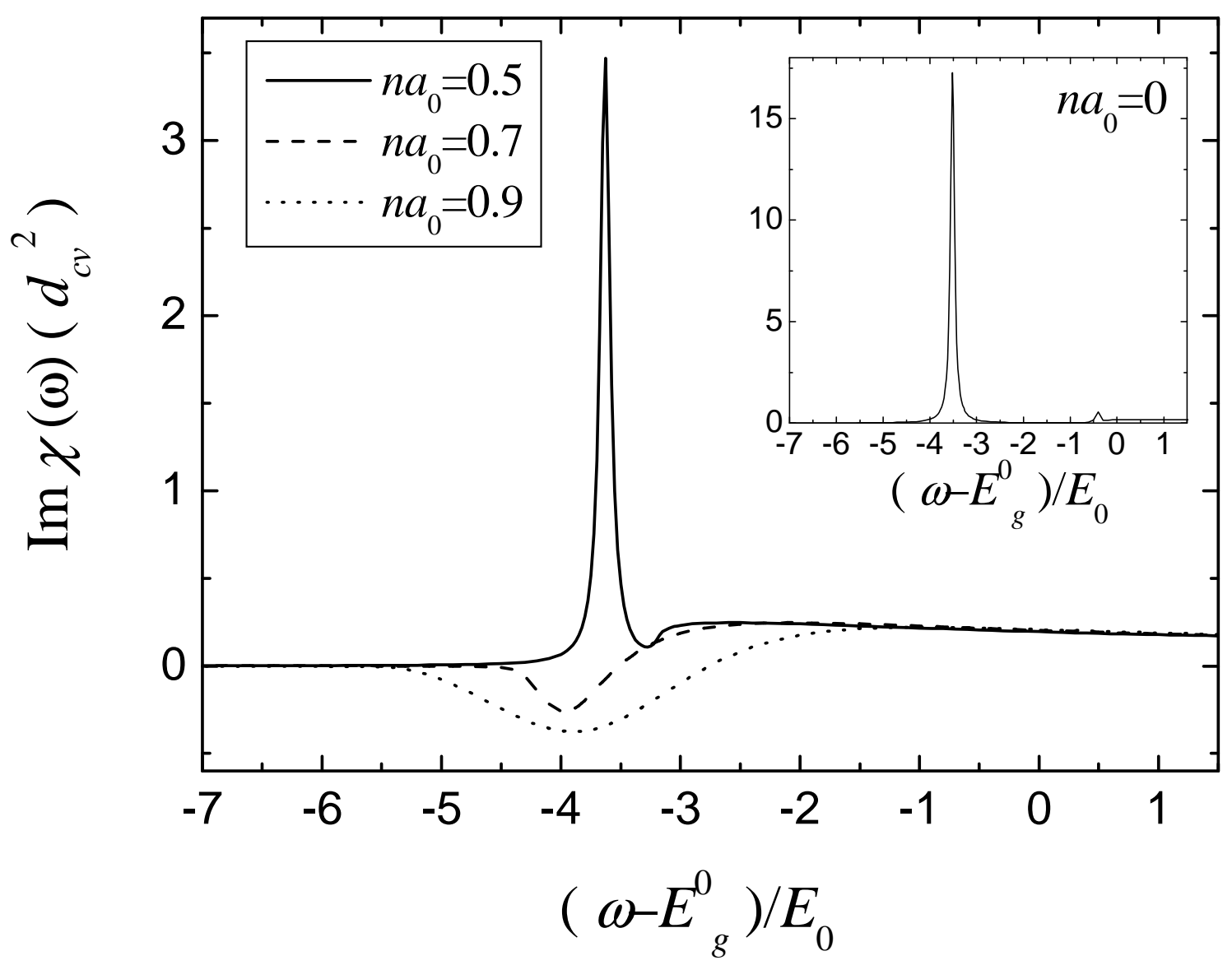

Fig.3 


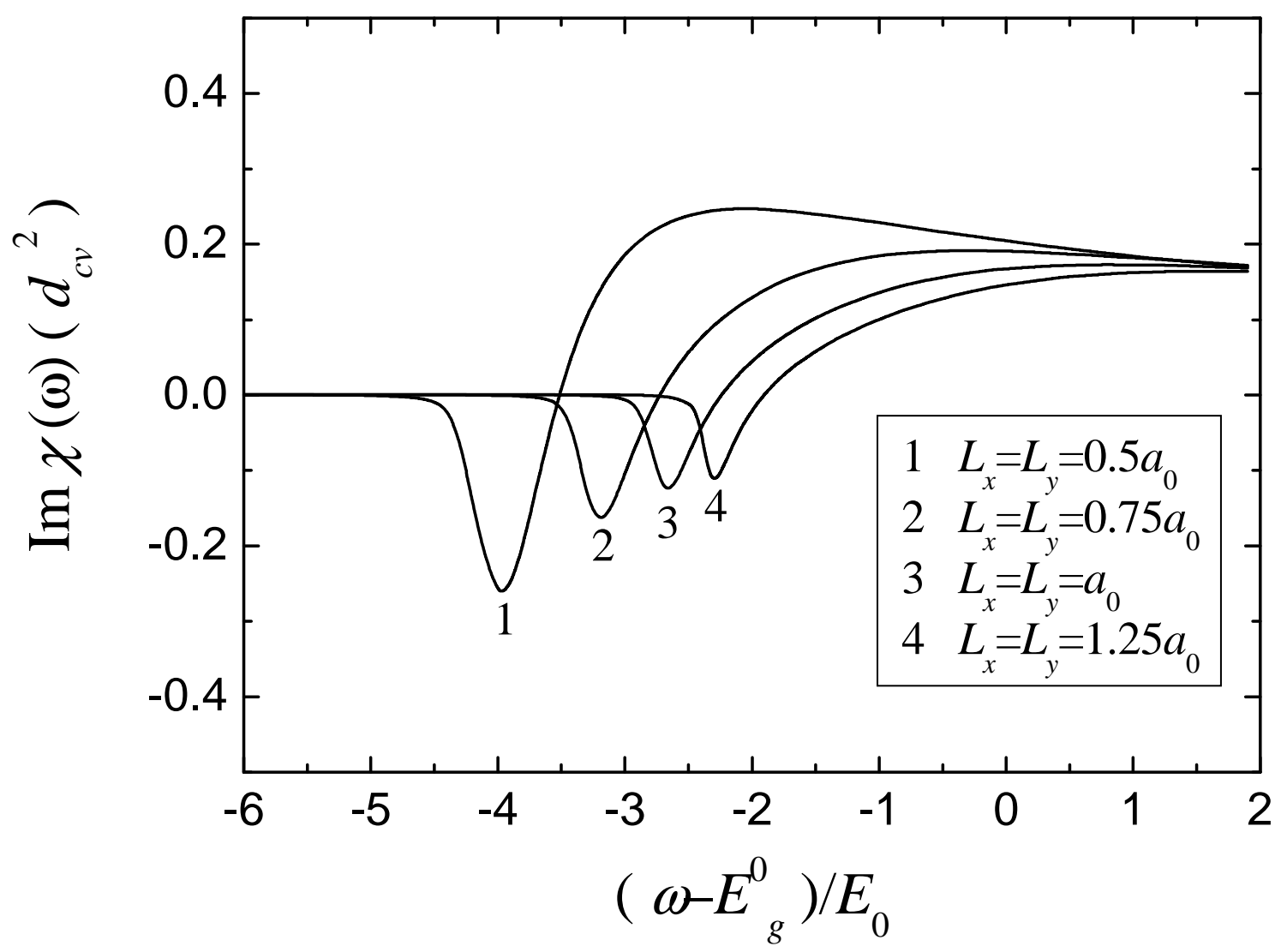

Fig.4 